\title{
The forgotten cause of stridor in the emergency department
}

This article was published in the following Dove Press journal:

Open Access Emergency Medicine

16 January 2017

Number of times this article has been viewed

\section{Tian-Tee $\mathrm{Ng}$}

Ear, Nose and Throat Unit, Department of Surgery, Frankston Hospital, Peninsula Health, Frankston, VIC, Australia
Correspondence: Tian-Tee Ng ENT Unit, Department of Surgery, Frankston Hospital, PO Box 52, Frankston 3199, VIC, Australia

Tel +6 I 426266890

Fax +6I 397847568

Email tntdynamites@yahoo.com
Abstract: Paradoxical Vocal Fold Movement Disorder is where the larynx exhibits paradoxical vocal cords closure during respiration, creating partial airway obstruction. Causes of vocal fold movement disorder are multifactorial, and patients describe tightness of throat, difficulty getting air in, have stridor, and do not respond to inhalers. We propose using transnasal laryngoscopy examination, which will show narrowing of vocal cords on inspiration, and The Pittsburgh Vocal Cord Dysfunction Index with a cutoff score of $\geq 4$ to distinguish vocal fold movement disorder from asthma and other causes of stridor. Management of paradoxical vocal fold movement disorder involves a combination of pharmacological, psychological, psychiatric, and speech training. Paradoxical vocal fold movement disorder is a very treatable cause of stridor, so long as it is identified and other organic causes are excluded.

Keywords: paradoxical vocal fold movement disorder, stridor, emergency

\section{Introduction}

When a patient with stridor presents to the emergency department (ED), the most probable differential diagnosis that comes to mind would be vocal cord paralysis, organic upper airway obstruction (tumor, abscess, infections, and allergic reaction), or a foreign body in the airway. An unusual, but possible, cause of stridor in these circumstances could be paradoxical vocal fold movement disorder (PVFMD). The aim of this article was to increase the awareness of PVFMD, particularly among doctors in the front line of health care.

PVFMD is a condition where the larynx exhibits intermittent, paradoxical closure of the vocal cords during respiration in someone with an otherwise normal vocal cord motion, thereby creating a partial extrathoracic airway obstruction. This condition is also known as paradoxical vocal cord dysfunction, paradoxical vocal fold motion, vocal cord dysfunction, episodic laryngeal dyskinesia, irritant vocal cord dysfunction, psychogenic and variable vocal cord dysfunction, transient laryngeal dyskinesia, fake asthma, and Munchausen's stridor. PVFMD was first described in the medical literature in 1842, in a patient with hysteric croup. The paradoxic movement itself was first visualized through laryngoscopy in 1869 by Mackenzie, who visualized glottic closure in direct correlation with the patient's stridor. ${ }^{1}$

PVFMD is more common than previously recognized and should be considered when dyspnea is present without pulmonary disease or is out of proportion to the 
degree of coexistent pulmonary disease. ${ }^{1}$ As Chevalier Jackson (an American pioneer in laryngology) often quoted, "not all that wheezes is asthma."2 PVFMD is often misdiagnosed as asthma, which leads to the inappropriate choice of treatment options for subjects with this condition. ${ }^{3}$ Undiagnosed PVFMD has been shown to lead to immense health care costs where there are reports of unnecessary intubations and tracheostomies performed in these cases ${ }^{1}$ and increased asthma medication usage. ${ }^{4}$ One study found that $42.4 \%$ of patients with PVFMD were misdiagnosed with asthma for, on average, 9 years. ${ }^{4}$ Although the exact prevalence of PVFMD is not known, it is estimated that the rate of incidence is between $2.5 \%$ and $26.9 \%{ }^{3}$ Young females and athletes have been reported to have a greater incidence of this disorder. ${ }^{3}$

\section{Etiology}

Initially, the cause of PVFMD was thought to be psychological, with stress and anxiety as the main triggers but this clinical picture has evolved and PVFMD is currently thought to be influenced more by medical causes than previously recognized. ${ }^{1}$ The exact physiological mechanism of PVFMD remains unknown. However, proposed theories include altered autonomic input from central brain regions polysynaptically linked to the larynx resulting in laryngeal hyper-responsiveness. Other possibilities include inflammation and/or irritation of the vocal cords ${ }^{4}$ or direct stimulation of the sensory nerve endings in the respiratory tract by noxious stimuli such as cold air, smoke, or other airborne environmental or occupational irritants ${ }^{5}$ and exertion as a trigger. In addition, hyperventilation, tobacco, allergic laryngitis, viral illness, untreated sleep apnea, gastroesophageal reflux disease, and rhinitis with postnasal drip have all been suggested to trigger PVFMD. ${ }^{1,4,6}$ Forrest et al proposed that PVFMD is a descriptive term that is multifactorial and the etiology should direct treatment. ${ }^{7}$ They proposed a classification scheme that divides PVFMD into primary (75\%), which is psychological, and secondary (25\%), which consists of medical disorders divided into irritable larynx syndrome and neurologic disorder. ${ }^{8}$ Typical episodes of PVFMD last from seconds to minutes and occur primarily but not exclusively during inspiration. They are felt in the upper respiratory tract, resulting in symptoms identical to upper airway obstruction such as cough, choking, and shortness of breath. ${ }^{3}$

\section{Presentation}

When PVFMD is suspected, it is important to complete a diagnostic workup to exclude organic causes such as respira- tory, cardiac, or other serious conditions that require urgent medical treatment. ${ }^{3}$ There are several symptoms and elements of the patient's history that are characteristic of PVFMD. These include a tight feeling in the throat, more difficulty getting air in than out, being unresponsive to inhalers, and symptoms that are precipitated by anxiety, strong emotion, odors, changes in humidity or temperature, or exposure to chemicals. ${ }^{1}$ Dyspnea both develops more rapidly with PVFMD than with asthma and resolves more quickly with rest. ${ }^{1}$ However, PVFMD may not be seen on examination, largely because it is intermittent and it can be difficult to conduct laryngoscopy examination in the middle of an acute attack because of patient distress. ${ }^{3}$ It has been suggested that laryngoscopy examination is invasive, uncomfortable, and may interfere with normal laryngeal function or even induce PVFMD. ${ }^{8}$ Nevertheless, until correlates of laryngeal closure are determined or noninvasive methods of inspecting the larynx are discovered, laryngoscopy remains the primary diagnostic tool. ${ }^{8}$ Thus, to diagnose PVFMD, we propose performing transnasal laryngoscopy examination and applying The Pittsburgh Vocal Cord Dysfunction Index. ${ }^{4}$ The latter is a useful clinical scoring system to help distinguish PVFMD from asthma.

\section{Transnasal laryngoscopy}

On laryngoscopy examination, vocal cords movement during respiration in patients without PVFMD typically shows wide abduction during inspiration and slight narrowing with exhalation. In contrast, patients with PVFMD show narrowing on inspiration and/or an even more marked narrowing on exhalation ${ }^{1}$ and adduction or prolapse of the supraglottic laryngeal structures. ${ }^{9}$ One of the classical hallmark findings is the complete adduction of the vocal cords except for a small posterior diamond-shaped glottic chink during inspiration (Figure 1). ${ }^{6} \mathrm{~A}$ recent study showed that up to $76.9 \%$ of patients (90 out of 117) with PVFMD had evidence of laryngeal edema. ${ }^{9}$ However, it is important to note that transnasal laryngoscopy can also fail to document these characteristic findings, even in patients who are known to have PVFMD. ${ }^{9}$ Therefore, provocative maneuvers, such as exertion or noxious stimuli, are commonly employed to increase the likelihood of capturing an episode of PVFMD during laryngoscopy.7,9

\section{Pittsburgh Vocal Cord Dysfunction Index}

The Pittsburgh Vocal Cord Dysfunction Index ${ }^{4}$ (Table 1) is the first scoring system developed for distinguishing PVFMD from asthma but it has limitations where it should not be 


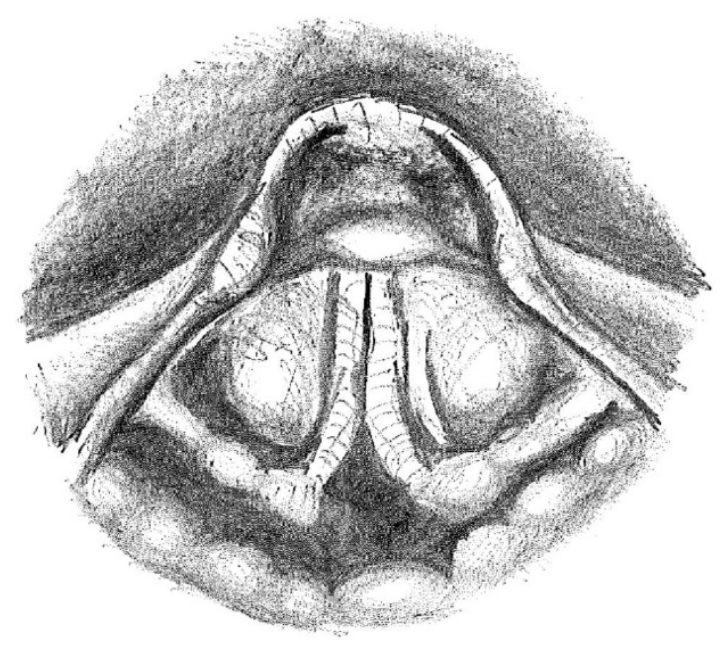

Figure I Typical adducted vocal cords with posterior glottic chink seen on laryngoscopy during stridor.

Table I Pittsburgh Vocal Cord Dysfunction Index

\begin{tabular}{ll}
\hline Symptoms & Score \\
\hline Dysphonia & 2 \\
Absence of wheezing & 2 \\
Throat tightness & 4 \\
Odors identified as a trigger & 3 \\
Maximum score=II & \\
\hline
\end{tabular}

Notes: Data from Traister et al. ${ }^{4}$

Table 2 Clinical features that should raise the possibility of paradoxical vocal fold movement disorder

Asthma-like symptoms that do not respond to bronchodilators or corticosteroids

Asthma-like symptoms that are precipitated by stress, emotional factors, or anxiety

Exercise-induced asthma

Athletes with choking sensation during exercise

Irritant-induced asthma-like symptoms

Note: Reproduced from Postgrad Med J, Paradoxical vocal cord motion disorder: past, present and future, Ibrahim WH, Gheriani HA, Almohamed AA, Raza T, 2007;83(977):164-172. ( 2007 The Fellowship of Postgraduate Medicine with permission from BMJ Publishing Group Ltd. ${ }^{6}$

used in a population of subjects with coexisting PVFMD and asthma. ${ }^{4}$ The cutoff score of 4 or more has a high predictive value for the presence of PVFMD, and coupled with a few questions (Table 2$)^{6}$ and no additional cost to the patient, may aid doctors in distinguishing PVFMD from asthma. ${ }^{4}$ Spirometric measurement of air flow volume is an additional diagnostic tool for PVFMD as it is minimally invasive. However, it may not be easily obtained in the ED. Therefore, we recommend that for diagnosis of PVFMD in the ED, a careful history of the condition must be taken plus completion of both laryngoscopy and the Pittsburgh Vocal Cord Dysfunction Index.

\section{Differential diagnosis}

The aim was to eliminate the differential diagnoses of PVFMD, such as bilateral vocal fold immobility, tracheal obstruction/ stenosis, neuromuscular disorder, asthma, exercised-induced asthma, congenital abnormalities, laryngeal collapse, subglottic mass/stenosis, croup, panic disorder, laryngeal tumor, foreign body aspiration, and bronchial obstruction.

\section{Acute management}

The acute management of PVFMD first requires establishing the correct diagnosis in patients during acute symptoms. ${ }^{2}$ Once diagnosis is confirmed, treatment should then be directed at acutely relieving airway obstruction. ${ }^{2}$ The first step is to reassure the patients that this condition is benign and that their oxygen levels are normal despite the severe dyspnea, while calmly quashing their fears. ${ }^{8}$ Kramer et al found that by educating and showing the patient normal vocal fold movements during respiration, followed by their own laryngoscopic video recordings with evidence of constriction during respiration, is often helpful. ${ }^{8}$ Morris et al found ample evidence of the effectiveness of relieving acute airway symptoms with reassurance alone. ${ }^{9}$ Various nonspeech tasks for the patient can open up the airway and possibly abort PVFMD attacks and include sniffing, pursed lip breathing on exhalation, and nasal inhalation. ${ }^{8}$ One of the most effective treatments for PVFMD is laryngeal control therapy focusing on relaxation techniques, comprehension of mental control over breathing, and stress reduction, which involves pulsed nasal inspiration (sniffs) with pursed lip expiration (blowing out candles) or through a straw. ${ }^{9}$ These techniques should be performed with the transnasal laryngoscope in situ to identify the most effective techniques for keeping the airway patent. ${ }^{9}$

Benzodiazepines can be used to sedate patients and relieve their anxiety, terminating the acute symptoms of PVFMD, but it is prudent to confirm normal oxygen saturation and exclude hyperpnea prior to administration. ${ }^{6}$ Heliox is a gaseous mixture of oxygen and helium and its use as a treatment has been shown to have an immediately favorable and often a sustained response after discontinuation. ${ }^{2}$ Heliox works because it is less dense than air; thus, inhalation reduces turbulence in the airway and eliminates respiratory noise, thus providing relief from dyspnea in patients with PVFMD and other forms of upper airway obstructive disease. ${ }^{6}$ Heliox itself does not relax the vocal folds, but relaxes the patient by decreasing the work of breathing, which then leads to relaxation of the vocal folds. ${ }^{8}$ Finally, treating with nebulized lignocaine has also demonstrated good symptomatic relief; hence, nebulized lignocaine may be trialed for relief of an acute attack. ${ }^{6}$ 


\section{Long-term management}

Long-term management of PVFMD involves a combination of pharmacological, psychological, psychiatric, and speech training. ${ }^{5}$ The associated goals include means of managing future exacerbations brought on by illness or stress, cessation of unnecessary asthma medication use, and avoidance of ED visits or missed work or school. ${ }^{1}$ Laryngeal control therapy (also called respiratory retraining therapy) with a speech pathologist is the cornerstone of treatment of PVFMD. ${ }^{1}$ Speech therapy plays an important role in long-term management by providing respiratory retraining. ${ }^{5}$ The role of speech therapy extends to assessment, diagnostic input, patient education, supportive counseling, PVFMD symptom management, and possible voice therapy. ${ }^{6}$ Attention to controlling anxiety and stress levels is important. However, the role of counseling or psychiatric care in treating PVFMD may decrease as the contribution of medical comorbidities becomes more widely recognized. ${ }^{1}$

\section{Conclusion}

PVFMD is a very treatable cause of stridor, so long as it is identified and other organic causes are excluded. ${ }^{1}$ Treatment for PVFMD requires a multidisciplinary approach as it is a multifactorial disease and is guided by the comorbidities present; hence, treatment plans must be tailored to each individual patient.

\section{Acknowledgments}

The author thanks Dr Vicky Tobin and Frankston Hospital Library. This project was not financially supported by any external sources and was fully funded by the Department of Surgery, Frankston Hospital.

\section{Disclosure}

The author reports no conflicts of interest in this work.

\section{References}

1. Matrka L. Paradoxic vocal fold movement disorder. OtolaryngolClin NAm. 2014;47(1):135-146.

2. Christopher KL, Morris MJ. Vocal cord dysfunction, paradoxic vocal fold motion, or laryngomalacia? Our understanding requires an interdisciplinary approach. OtolaryngolClin N Am. 2010;43(1):43-66.

3. Frank-Ito DO, Schulz K, Vess G, Witsell DL. Changes in aerodynamics during vocal cord dysfunction. ComputBiol Med. 2015;57:116-122.

4. Traister RS, Fajt ML, LandsittelD, Petrov AA. A novel scoring system to distinguish vocal cord dysfunction from asthma. JAllergy Clin Immunol Pract. 2014;2(1):65-69.

5. Chiang WC, Goh A, Ho L, Tang JP, Chay OM. Paradoxical vocal cord dysfunction: when a wheeze is not asthma. Singapore Med J. 2008;49(4): $110-112$.

6. Ibrahim WH, Gheriani HA, Almohamed AA, Raza T. Paradoxical vocal cord motion disorder: past, present and future. Postgrad Med J. 2007; 83(977):164-172.

7. Forrest LA, Husein T, Husein O. Paradoxical vocal cord motion: classification and treatment. Laryngoscope. 2012;122(4):844-853.

8. Kramer S, deSilva B, Forrest LA, Matrka L. Does treatment of paradoxical vocal fold movement disorder decrease asthma medication use? Laryngoscope. Epub 2016 Nov 15.

9. Hicks M, Brugman SM, Katial R. Vocal cord dysfunction/paradoxical vocal fold motion. Prim Care. 2008;35(1):81-103.
Open Access Emergency Medicine

\section{Publish your work in this journal}

The Open Access Emergency Medicine is an international, peerreviewed, open access journal publishing original research, reports, editorials, reviews and commentaries on all aspects of emergency medicine. The manuscript management system is completely online and includes a very quick and fair peer-review system, which is all

\section{Dovepress}

easy to use. Visit http://www.dovepress.com/testimonials.php to read real quotes from published authors. 